\title{
Space-Time-Frequency Shift Keying for Dispersive Channels
}

\author{
Hoang Anh Ngo, Chao Xu, Shinya Sugiura, Member, IEEE, and Lajos Hanzo, Fellow, IEEE
}

\begin{abstract}
Inspired by the concept of the Space-Time Shift Keying (STSK) modulation, in this paper we proposed the Space-Frequency Shift Keying (SFSK) modulation as well as the Space-Time-Frequency Shift Keying (STFSK) concept which spreads the transmit signal not only across the space and time domains, but also the frequency domain. The performance of STSK modulation is degraded by about $2 \mathrm{~dB}$, when the channel changes from uncorrelated frequency-flat fading to the frequency-selective environment of the 6-tap COST207 model. By contrast, as a benefit of Frequency Shift keying, the SFSK and STFSK schemes are capable of maintaining their performance also in frequency-selective fading environments. Finally, we demonstrate that the STSK and SFSK schemes constitute special cases of the STFSK modulation.
\end{abstract}

Index Terms - Dispersive channels, diversity, FSK, space-timefrequency processing .

\section{INTRODUCTION}

$\mathbf{T}$ HE family of multiple-input-multiple-output (MIMO) arrangements designed for wireless communications has attracted substantial research attention owing to its potential to increase the attainable capacity without requiring additional bandwidth. The class of spatial division multiplexing, such as the family of BLAST schemes [1], is capable of increasing the transmission rate, i.e., the multiplexing gain, at the cost of significantly increasing the decoding complexity. By contrast, the low complexity Space-Time-Block-Codes (STBC) [2], [3] are capable of maximizing the attainable diversity order, but fail to attain a multiplexing gain. A tradeoff between the two schemes can be achieved by employing the Linear Dispersion Codes (LDC) proposed in [4].

Furthermore, Haas and his team [5] proposed the Spatial Modulation (SM), while the team of Ghrayaeb and Szczecinski [6] introduced the Space Shift Keying (SSK) concept into MIMO communications. The philosophy of these schemes is to activate only a single transmit antenna at any instant in order to maintain a low complexity, whilst avoiding any inter-antenna interference and inter-antenna synchronization. Motivated by the above concepts, the authors of [7] conceived Space-Time Shift Keying (STSK), which strikes an improved diversity versus multiplexing tradeoff. Furthermore, it results

Manuscript received November 01, 2010; revised January 04, 2011; accepted January 09, 2011. Date of publication January 17, 2011; date of current version January 27, 2011. This work was supported by the European Union's Seventh Framework Programme (FP7/2007-2013) under Grant 214625, the RC-UK under the auspices of the UK-India Advanced Technology Centre, and by the China-UK Science Bridge in 4G Wireless Communications. The associate editor coordinating the review of this manuscritp and approving it for publication was Dr. Yuriy V. Zakharov.

The authors are with the School of Electronics \& Computer Science, University of Southampton SO17 1BJ, U.K. (e-mail: han08r@ecs.soton.ac.uk; cx1g08@ecs.soton.ac.uk; ss07r@ecs.soton.ac.uk; lh@ecs.soton.ac.uk; http://www-mobile.ecs.soton.ac.uk).

Digital Object Identifier 10.1109/LSP.2011.2106773 in a reduced-complexity system operating at a higher capacity than the SM/SSK and BLAST schemes.

Against this backcloth, the novel contribution of this paper is that we propose the Space-Frequency Shift Keying (SFSK) concept, where the transmit signal is spread across both the space and frequency domains, as well as the Space-Time-Frequency Shift Keying (STFSK) concept, where a beneficial diversity gain may be gleaned from three different domains, namely the space-, time-and frequency-domain. In addition to the advantages provided by STSK modulation, the STFSK scheme also avoids the Inter-Symbol Interference (ISI) imposed by frequency-selective fading channels.

The outline of this letter is as follows. Section II describes the system's architecture and the associated assumptions, followed by the corresponding performance results in Section III. Finally, our concluding remarks are offered in Section IV.

\section{Space-Time-Frequency ShIFT Keying Modulation}

In this contribution we consider an $(M \times N)$-element system, where the transmitter and receiver employ $M$ and $N$ antennas, respectively. The channel is assumed to impose frequency-selective Rayleigh fading. Generally, a transmission block-based system model may be described as

$$
\mathbf{Y}(i)=\sum_{j=0}^{J-1} \mathbf{H}(i-j, j) \mathbf{S}(i-j)+\mathbf{V}(i),
$$

where $i$ indicates the block index and $j$ implies the tap index in the tap-delay-line channel model, which consists of $J$ taps. It is noted that flat fading in encountered for $J=1$. Furthermore, $\mathbf{Y} \in C^{N \times T}$ represents the received signals of the $N$ antennas and $\mathbf{S} \in C^{M \times T}$ denotes the signal transmitted from the $M$ antennas. Furthermore, $\mathbf{H} \in C^{N \times M}$ characterizes the communication channels, each obeying correlated frequency-selective Rayleigh fading. Finally, $\mathbf{V}$ denotes the complex-valued zero-mean Gaussian distribution of $C N\left(0, N_{0}\right)$, where $N_{0}$ is the noise variance. It is also assumed that fading and noise coefficients remain constant during each time slot.

Let us now briefly review the STSK modulation proposed in [7]. Moreover, we introduce two novel modulation schemes, namely the SFSK as well as the STFSK regimes, where the signal is spread across the space-time-frequency domain. The normalized throughput expressions of the three schemes are provided at the end of this section.

\section{A. Space-Time Shift Keying}

Fig. 1 illustrates the transmitter of the STSK scheme, where the information bits are divided into two parallel bits streams. The first bit stream is mapped by $Q$ predefined dispersion matrices $\mathbf{A}_{q} \in C^{M \times T}(q=1,2, \ldots, Q)$ [8], while the second is mapped to $s_{l}(i)$ symbols $(l=1,2, \ldots, L)$ by a conventional 


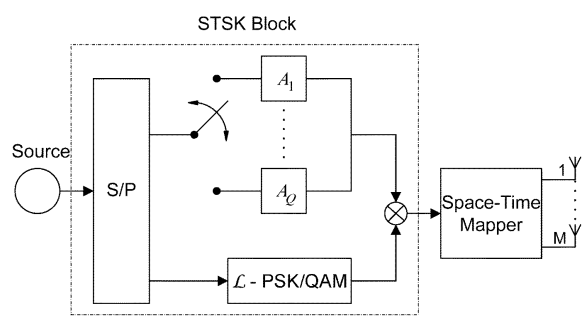

Fig. 1. Transmitter block diagram of STSK scheme.

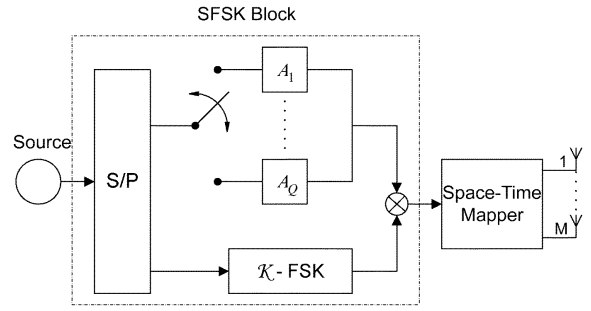

Fig. 2. Transmitter block diagram of SFSK scheme.

modulation scheme, such as $L$-PSK or $L$-QAM. Then, the resultant streams are multiplied together in order to create the space-time block $\mathbf{S}(i) \in C^{M \times T}$, which consists of a total of $\log _{2}(Q . L)$ source bits, yielding

$$
\mathbf{S}(i)=s(i) \mathbf{A}(i) \text {. }
$$

It is noted that each symbol $\mathbf{S}(i)$ is a function of time during the period $T_{s}$ of each time slot. As in [7], the signal received at the destination may be presented in scalar form as

$$
\overline{\mathbf{Y}}(i)=\overline{\mathbf{H}}(i) \chi \mathbf{K}(\mathbf{i})+\overline{\mathbf{V}}(i)
$$

with the variables formulated as

$$
\begin{aligned}
\overline{\mathbf{Y}}(i) & =\operatorname{vec}(\mathbf{Y}(i)) \in \mathcal{C}^{N T \times 1}, \\
\overline{\mathbf{H}}(i) & =\mathbf{I} \otimes \mathbf{H}(i) \in \mathcal{C}^{N T \times M T}, \\
\overline{\mathbf{V}}(i) & =\operatorname{vec}(\mathbf{V}(i)) \in \mathcal{C}^{N T \times 1}, \\
\chi & =\left[\operatorname{vec}\left(\mathbf{A}_{1}\right) \cdots \operatorname{vec}\left(\mathbf{A}_{Q}\right)\right] \in \mathcal{C}^{M T \times Q}
\end{aligned}
$$

where $\mathbf{I}$ is the $(T \times T)$-element identity matrix and $\otimes$ is the Kronecker product. Furthermore, $\mathbf{K}(i) \in C^{Q \times 1}$ is the equivalent transmitted signal vector, which may be expressed as

$$
\mathbf{K}(i)=[\underbrace{0, \cdots, 0}_{q-1}, s(i), \underbrace{0, \cdots, 0}_{Q-q}]^{T r}
$$

where $\operatorname{Tr}$ indicates the matrix transpose operation.

When the coherent maximum likelihood detector [9], [10] is employed, the STSK modulated signal may be recovered by searching for an appropriate pair of the $l^{\text {th }}(l=1, \cdots, L)$ PSK/QAM symbol and the $q^{t h}(q=1, \cdots, Q)$ dispersion matrix. More particularly, the estimate $(\hat{q}, \hat{l})$ is given by minimizing the following metric

$$
\begin{aligned}
(\hat{q}, \hat{l})=\arg \min _{q, l} \| & \overline{\mathbf{Y}}(i)-\overline{\mathbf{H}}(i) \chi \mathbf{K}_{q, l}(i) \\
& -\sum_{j=1}^{J-1} \overline{\mathbf{H}}(i-j, j) \chi \mathbf{K}(i-j) \|^{2}
\end{aligned}
$$

$$
\begin{aligned}
=\arg \min _{q, l} \| & \overline{\mathbf{Y}}(i)-s_{l}(i)(\overline{\mathbf{H}}(i) \chi)_{q} \\
& -\sum_{j=1}^{J-1} \overline{\mathbf{H}}(i-j, j) \chi \mathbf{K}(i-j) \|^{2}
\end{aligned}
$$

where $s_{l}(i)$ is the $l^{t h}$ symbol in the $L$-point constellation at the $i^{\text {th }}$ block index and the signal vector $\mathbf{K}_{q, l} \in K(1 \leq q \leq$ $Q, 1 \leq l \leq L)$ is presented by

$$
\mathbf{K}_{q, l}(i)=[\underbrace{0, \cdots, 0}_{q-1}, s_{l}(i), \underbrace{0, \cdots, 0}_{Q-q}]^{T r} .
$$

Furthermore, $\sum_{j=1}^{J-1} \overline{\mathbf{H}}(i-j, j) \chi \mathbf{K}(i-j)$ represents the delayed paths of the dispersive channel, which is omitted in flat-fading environment while $(\mathbf{H}(i) \chi)_{q}$ denotes the $q$ th column of the ma$\operatorname{trix}(\mathbf{H}(i) \chi)$.

It is noted that $M$ should be less than or equal to $T(M \leq T)$, since no further capacity gain may be achieved for $M>T$, as shown in [11], [12]. Additionally, in order to maintain a unity transmission power for a STSK symbol duration, each of the $Q$ dispersion matrices has to obey the power constraint of [7]

$$
\operatorname{tr}\left[\mathbf{A}_{q}^{\dagger} \mathbf{A}_{q}\right]=T \quad(q=1, \cdots, Q)
$$

where $\operatorname{tr}[\cdot]$ indicates the trace operation, while the superscript $\dagger$ denotes the complex conjugate transpose operation.

\section{B. Space-Frequency Shift Keying}

The transmitter of the SFSK modulation scheme is shown in Fig. 2, where the PSK/QAM modulator is replaced by the FSK modulator. Due to this modification, the transmitted block $\mathbf{S}(i) \in C^{M \times T}$ is modified as

$$
\mathbf{S}(i)=r(i) \mathbf{A}(i)
$$

where the $\log _{2}(K)$ source bits are mapped to the FSK symbol $r(i)$, which is represented by

$$
r(i)=\cos \left(2 \pi f_{i} t+\varphi_{i}\right) .
$$

In order to maintain orthogonality, the frequency between the adjacent signalling tones of the FSK modulator has to be separated by at least $1 / T_{s} \mathrm{~Hz}$, where $T_{s}$ is the duration of a time slot

At the receiver, we employ a demodulator consisting of a bank of $K$ parallel square-law detectors in order to detect the activated frequencies of the SFSK symbols. Then the ML detector is employed to identify the transmitted dispersion matrix by substituting

$$
\mathbf{K}_{q}(i)=[\underbrace{0, \cdots, 0}_{q-1}, r(i), \underbrace{0, \cdots, 0}_{Q-q}]^{T r}
$$

into (9)

In order to maintain orthogonality, the frequency between the adjacent signalling tones of the FSK modulator has to be separated by at least $1 / T_{s} \mathrm{~Hz}$, where $T_{s}$ is the symbol period .

\section{Space-Time-Frequency Shift Keying}

Based on the STSK and SFSK modulation schemes, the architecture of the STFSK scheme is portrayed in Fig. 3, where 


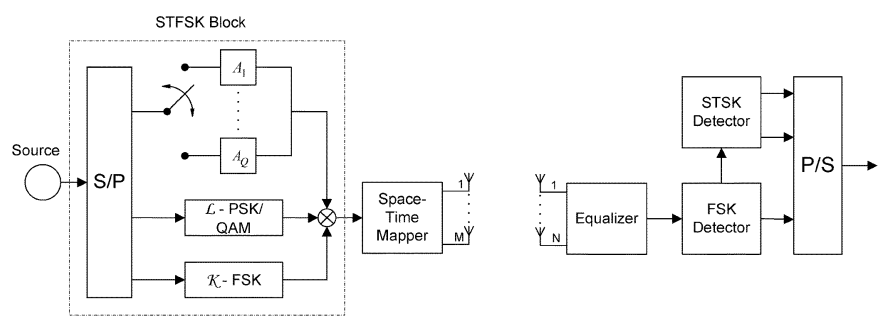

Fig. 3. The transceiver block diagram of STFSK scheme.

three symbol sub-streams are combined before being mapped to the transmit antennas. As a result, the transmitted signal may be expressed as

$$
\mathbf{S}(i)=r(i) s(i) \mathbf{A}(i)
$$

Again, a low-complexity square-law detector is employed first to detect the activated frequencies, i.e the FSK symbols. Then, the PSK/QAM symbols and the dispersion matrices are detected by substituting the following vector into (9)

$$
\mathbf{K}_{q, l}(i)=[\underbrace{0, \cdots, 0}_{q-1}, r(i) s_{l}(i), \underbrace{0, \cdots, 0}_{\begin{array}{c}
Q-q \\
(1 \leq q \leq 0
\end{array}}]^{T r}
$$

It may be observed that the STSK and the SFSK schemes constitute special cases of STFSK, when we have $K=1$ and $L=1$, respectively. In order to reduce the impact of the ISI imposed by frequency-selective fading, the signal may be equalized before carrying out detection.

\section{Throughput}

1) STFSK: For an STFSK system activating $K$ orthogonal frequencies, each STFSK block consists of $\log _{2}(K L Q)$ bits, which are transmitted during $T$ symbol periods. Hence, the normalized throughput of STFSK may be expressed as

$\eta_{S T F S K}=\frac{2 \log _{2}(K L Q)}{K T} \quad\left(\mathrm{bits} / \mathrm{T}_{s} /\right.$ frequency).

2) STSK: An STSK system may be considered as a simplified STFSK arrangement having a single transmit frequency $(K=1)$. Therefore, the normalized throughput of STSK may be expressed as

$$
\eta_{S T S K}=\frac{\log _{2}(L Q)}{T} \quad\left(\text { bits } / \mathrm{T}_{s} / \text { frequency }\right) .
$$

It is noted that (19) is identical to (3) of [7].

3) SFSK: Similarly, an SFSK scheme may be considered as a simplified STFSK system employing $K$ transmit frequencies and a single-symbol PSK/QAM modulation scheme ( $L=1$ ). Therefore, the normalized throughput of SFSK may be calculated as

$$
\eta_{S F S K}=\frac{2 \log _{2}(K Q)}{K T} \quad\left(\text { bits } / \mathrm{T}_{s} / \text { frequency }\right) \text {. }
$$

\section{PARAMETER SELECTION}

The STFSK is a sophisticated scheme, which beneficially transforms the binary bits into the space-time-frequency-domain signal. Therefore, appropriately configuring the system parameters is important.
For a system having a relatively narrow bandwidth and operating in a flat-fading channel, STSK, which represents STFSK associated with $K=1$, constitutes a suitable selection. This is because FSK only exhibits substantial advantages in frequencyselective fading channels, where transmission during prolonged fades are avoided by hopping to an independently faded tone outside the coherence bandwidth. Moreover, a tight bandwidth constraint does not allow a sufficiently high number of frequencies to be selected. The parameter configuration process for the STSK scheme was detailed in [7].

By contrast, when a sufficiently high bandwidth is available, the STFSK concept $(K \geq 2)$ may significantly improve the attainable system performance, as a benefit of spreading the signal over the space-time-frequency domain. Moreover, it can also offer a high system configuration flexibility. Let us briefly exemplify the mapping rules of our STFSK scheme, where a fixed number of $B=\log _{2}(K L Q)=3$ bits per space-time-frequency block $S$ is transmitted. We can use one of the following combinations:

$$
\begin{aligned}
& B=\log _{2}(K L Q)=3 \text { bits } \\
& \Leftrightarrow\left\{\begin{array}{l}
K=1,(L, Q)=(1,8 ; 2,4 ; 4,2 ; 8,1) \\
K=2,(L, Q)=(1,4 ; 2,2 ; 4,1) \\
K=4,(L, Q)=(1,2 ; 2,1) \\
K=8,(L, Q)=(1,1) .
\end{array}\right.
\end{aligned}
$$

Clearly, for a fixed number of 3 bits per transmitted block, the STFSK scheme may offer ten different system configuration choices, compared to the four STSK options of [7].

According to (18), as the value of $K$ or $T$ increases, the throughput of the system linearly decreases. At the same time, the throughput is increased relatively slowly, namely as a function of $\log _{2}(K \cdot L \cdot Q)$ with the product $(K \cdot L \cdot Q)$. Again, the classic tradeoff between the achievable diversity and multiplexing gain has to be considered here. Moreover, increasing $M$ also leads to an increased complexity at the demodulator, since more matched filters are required. At the same time, the BER improvement slows down for high values of $M$, namely for $M>4$. Therefore, the values of $M=\{2,4\}$ constitute reasonable choices in order to mitigate the effects of frequency-selective fading, while maintaining a high throughput. Finally, the number of transmit antennas employed should obey $M \leq T$, as mentioned above.

\section{PERFORMANCE RESUlts}

In this section, the BER performance of the STSK, SFSK and STFSK modulation schemes will be investigated and compared. The modulation parameters of the three schemes are provided in Table I, where the normalized throughput of $1 \mathrm{bit} / T_{s} /$ frequency is applied to all schemes in order to make a fair comparison. At the receiver, the square-law detector is employed to detect the FSK symbols while the maximum likelihood STSK detector is utilized to detect the PSK/QAM symbols and the LDC matrices.

Fig. 4 shows the BER performance of the three schemes, when channels are assumed to be uncorrelated and frequency-flat Rayleigh faded, where STSK achieves a power gain of about $3 \mathrm{~dB}$ over SFSK across the $E_{b} / N_{0}$ range considered. This may be explained by the fact that STSK is not affected by the frequency-flat fading. As an additional benefit, it may glean a diversity gain from the time domain, which SFSK fails to achieve. Hence, STSK may outperform SFSK. However, its BER performance is still worse than that of STFSK. More particularly, STFSK offers a power gain of $3 \mathrm{~dB}$ over STSK. 
TABLE I

MOdULATION SCHEMES' PARAMETERS

\begin{tabular}{|l|c|l|l|c|}
\hline Parameters & $M / N / T / Q$ & \multicolumn{1}{c|}{$L$} & $K$ & Throughput \\
\hline \hline STSK & $4 / 1 / 4 / 4$ & 4 & N/A & 1 bit $/ T_{s} /$ freq \\
\hline SFSK & $4 / 1 / 4 / 8$ & N/A & 2 & 1 bit/Ts $/$ freq \\
\hline STFSK & $4 / 1 / 4 / 2$ & 4 & 2 & 1 bit $/ T_{s} /$ freq \\
\hline
\end{tabular}

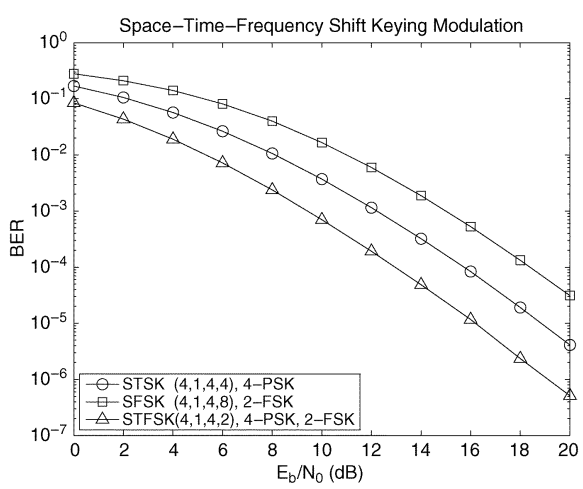

Fig. 4. Performance of the STSK, the SFSK, and the STFSK modulation schemes under the uncorrelated frequency-flat Rayleigh fading channels.

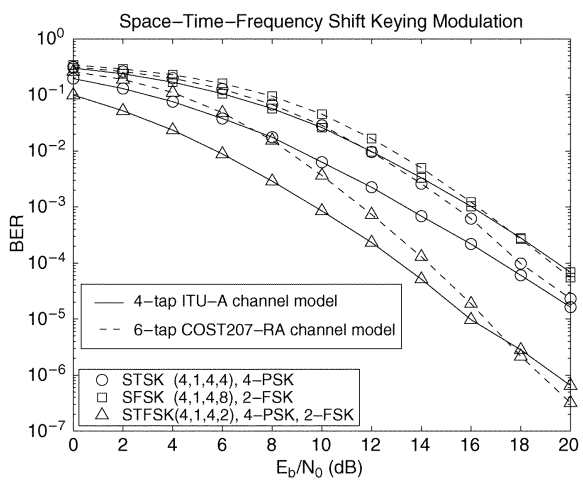

Fig. 5. Performance of the STSK, the SFSK, and the STFSK modulation schemes under the frequency-selective Rayleigh fading channel with the normalized Doppler frequency of 0.001 .

This may be explained by observing that STFSK transmits $Q=2$ symbols in $T=4$ time slots, compared to the $Q=4$ symbols transmitted in $T=4$ time slots by the STSK scheme. Therefore, STFSK is capable of gleaning a higher diversity gain from the time domain.

The performance of the three schemes in the frequency-selective fading environment considered is characterized in Fig. 5. The 4-tap ITU-A channel model [13] having a normalized Doppler frequency of 0.001 is employed here. As shown in the figure, STFSK outperforms the STSK and SFSK schemes. It is also observed that the performance of the STFSK and the SFSK systems remains more-or-less unaffected in the face of the frequency-selective fading channels considered, while the STSK's performance degrades by $1 \mathrm{~dB}$, since the STSK scheme attains no diversity gain in the frequency domain. Therefore, the STSK signal is contaminated by the ISI imposed by the frequency-selective fading.

Finally, we investigate the scenario when the ISI imposes a more grave impact on the attainable system performance by introducing the 6-tap COST207 rural area channel model [14]. According to Fig. 5, the performance of the STFSK and the STSK schemes recorded for the 6-tap COST207 channel be- comes more degraded in the low $E_{b} / N_{0}$ region, namely below $10 \mathrm{~dB}$, than for the 4-tap ITU-A channel but in the rest of the $E_{b} / N_{0}$ region, they perform comparably well. By contrast, the STSK's performance continues to degrade. More particularly, the gap between the BER curves of the STSK and the SFSK schemes is reduced by $2 \mathrm{~dB}$, compared to the uncorrelated frequency-flat fading scenario, resulting in a power gain of $1 \mathrm{~dB}$.

In conclusion, motivated by the recent concept of the STSK modulation scheme, in this contribution we proposed the generalized STFSK arrangement, which significantly improves the attainable system performance by dispensing the signal across the spatial domain, time domain as well as frequency domain. Our performance results showed that STFSK may offer a better performance than the STSK and SFSK schemes, regardless, whether the channel is frequency-selective or frequency-flat faded. More particularly, for transmission over the 6-tap COST207 channel model, STFSK achieves gains of $5 \mathrm{~dB}$ and $6 \mathrm{~dB}$ over STSK and SFSK, respectively, as shown in Fig. 5 at the BER of $10^{-4}$. Additionally, STSK suffered from the ISI imposed by the frequency-selective fading channel, requiring a $2 \mathrm{~dB}$ power boost when changing the channel model from uncorrelated flat-fading to the 6-tap COST207 rural area model. Finally, we also concluded that STSK and SFSK constitute special cases of STFSK.

\section{REFERENCES}

[1] G. J. Foschini, "Layered space-time architecture for wireless communication in a fading environment when using multi-element antennas," Bell Lab. Tech. J., vol. 1, pp. 41-59, 1996.

[2] S. M. Alamouti, "A simple transmit diversity technique for wireless communications," IEEE J. Sel. Areas Commun., vol. 16, no. 8, pp. 1451-1458, 1998.

[3] V. Tarokh, N. Seshadri, and A. R. Calderbank, "Space-time codes for high data rate wireless communication: Performance criterion and code construction," IEEE Trans. Inf. Theory, vol. 44, pp. 744-765, 1998.

[4] B. Hassibi and B. M. Hochwald, "High-rate codes that are linear in space and time," IEEE Trans. Inf. Theory, vol. 48, no. 7, pp. 1804-1824, 2002.

[5] R. Y. Mesleh, H. Haas, S. Sinanovic, C. W. Ahn, and S. Yun, "Spatial modulation," IEEE Trans. Veh. Technol., vol. 57, no. 4, pp. 2228-2241, 2008.

[6] J. Jeganathan, A. Ghrayeb, L. Szczecinski, and A. Ceron, "Space shift keying modulation for MIMO channels," IEEE Trans. Wireless Commun., vol. 8, no. 7, pp. 3692-3703, 2009.

[7] S. Sugiura, S. Chen, and L. Hanzo, "Coherent and differential space-time shift keying: A dispersion matrix approach," IEEE Trans. Commun., no. 11, pp. 3219-3230, Nov. 2010.

[8] M. Jiang and L. Hanzo, "Unitary linear dispersion code design and optimization for mimo communication systems," IEEE Signal Process. Lett., vol. 17, no. 5, pp. 497-500, 2010.

[9] J. Jeganathan, A. Ghrayeb, and L. Szczecinski, "Spatial modulation: Optimal detection and performance analysis," IEEE Commun. Lett., vol. 12 , no. 8 , pp. $545-547,2008$.

[10] S. Chen, S. Sugiura, and L. Hanzo, "Semi-blind joint channel estimation and data detection for space-time shift keying systems," IEEE Signal Process. Lett., vol. 17, no. 12, pp. 993-996, Dec. 2010.

[11] T. L. Marzetta and B. M. Hochwald, "Capacity of a mobile multipleantenna communication link in Rayleigh flat fading," IEEE Trans. Inf. Theory, vol. 45, no. 1, pp. 139-157, 1999.

[12] B. M. Hochwald and W. Sweldens, "Differential unitary space-time modulation," IEEE Trans. Commun., vol. 48, no. 12, pp. 2041-2052, 2000.

[13] Guidelines for the Evaluation of Radio Transmission Technologies for IMT-2000 1997.

[14] M. Patzold, Mobile Fading Channels: Modelling, Analysis and Simulation. New York: Wiley, 2001. 